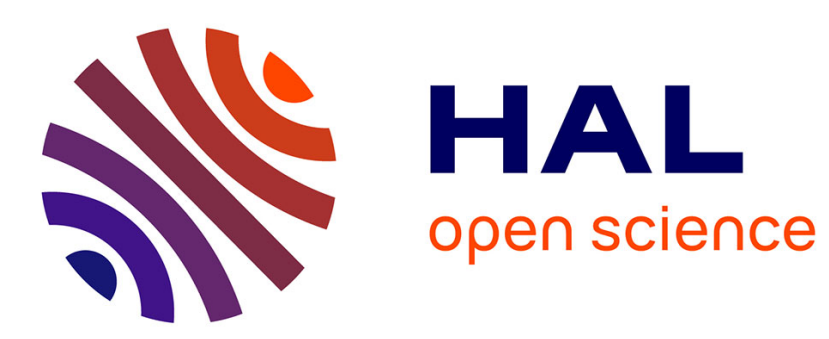

\title{
Distribution of Busy Period in Stochastic Fluid Models
}

Nelly Barbot, Bruno Sericola, Miklós Telek

\section{To cite this version:}

Nelly Barbot, Bruno Sericola, Miklós Telek. Distribution of Busy Period in Stochastic Fluid Models.

[Research Report] RR-4057, INRIA. 2000. inria-00072579

\section{HAL Id: inria-00072579 \\ https://hal.inria.fr/inria-00072579}

Submitted on 24 May 2006

HAL is a multi-disciplinary open access archive for the deposit and dissemination of scientific research documents, whether they are published or not. The documents may come from teaching and research institutions in France or abroad, or from public or private research centers.
L'archive ouverte pluridisciplinaire HAL, est destinée au dépôt et à la diffusion de documents scientifiques de niveau recherche, publiés ou non, émanant des établissements d'enseignement et de recherche français ou étrangers, des laboratoires publics ou privés. 


\section{Distribution of Busy Period in Stochastic Fluid Models}

Nelly Barbot, Bruno Sericola and Miklós Telek

\section{$\mathbf{N}^{\circ} \mathbf{4 0 5 7}$}

Novembre 2000

THÈME 1 



\title{
Distribution of Busy Period in Stochastic Fluid Models*
}

\author{
Nelly Barbot ${ }^{\dagger}$, Bruno Sericola ${ }^{\dagger}$ and Miklós Telek ${ }^{\ddagger}$ \\ Thème 1 - Réseaux et systèmes \\ Projet Armor
}

Rapport de recherche $\mathrm{n}^{\circ} 4057$ - Novembre 2000 - 23 pages

\begin{abstract}
We consider the busy period in a stochastic fluid flow model with infinite buffer where the input and output rates are controlled by a finite homogeneous Markov process. We derive an explicit expression for the distribution of the busy period and we obtain an algorithm to compute it which exhibits nice numerical properties.
\end{abstract}

Key-words: Stochastic fluid model, busy period, Markov process, numerical analysis.

(Résumé : tsvp)

* This work was supported by the French-Hungarian bilateral R\&D program 2000/2001.

$\dagger\{$ Nelly.Barbot $\}\{$ Bruno.Sericola\}@irisa.fr

${ }^{\ddagger}$ Dept. of Telecom., Technical University of Budapest, 1521 Budapest, Hungary, telek@hit.bme.hu 


\section{Distribution de la période d'occupation dans les modèles stochastiques fluides}

Résumé : On considère la période d'occupation dans un modèle stochastique fluide à capacité infinie où les taux d'entrée et de sortie sont contrôlés par un processus de Markov fini et homogène. On aboutit à une expression explicite de la distribution de la période d'occupation et on obtient un algorithme pour la calculer qui possède de bonnes propriétés numériques.

Mots-clé : Modèle stochastique fluide, période d'occupation, processus de Markov, analyse numérique. 


\section{Introduction}

Stochastic fluid models (SFM) are widely applied to capture the queueing behaviour of packet switched networks with large buffers [5]. An SFM is composed by a buffer and a background process that modulates the rate of the fluid accumulation in the buffer. The modulating process is commonly assumed to be a continuous time Markov chain (CTMC).

The transient analysis of SFMs, i.e., the analysis of fluid distribution in the buffer at time $t$, is a complex and computationally intensive task. The cardinality of the problem is characterized by the number of states of the modulating process. The majority of the published analysis approaches requires the spectral decomposition of a matrix of size of the state space $[4,2]$. The applicability of this approach is limited by the computational complexity and potential numerical instability due to close eigenvalues. In some special cases it is possible to obtain an analytical solution exploiting the special behaviour of the modulating process [1]. For general modulating processes a numerically stable recursive method was proposed in [6].

There are other important transient measures of SFMs that are considered in the literature. The importance of the distribution of the busy period ${ }^{1}$ was introduced in [3], where a SFM with two priorities is studied. In the considered model the higher priority stream occupies the server capacity as long as there is "high priority fluid" in the buffer and the low priority stream gets service only when there is no high priority fluid in the buffer. The low priority stream is served with server vacation, where the server vacation is the busy period of the high priority stream.

In this paper we provide a stable numerical method to evaluate the distribution of the busy period in SFMs with infinite capacity. The remainder of the paper is organized as follows. Section 2 provides the proposed numerical procedure, while Section 3 introduces a numerical example.

\section{Stochastic fluid models}

Let $\{Z(t), t \geq 0\}$ be an irreducible CTMC on a finite state space $S$ with generator $A=\left[a_{i j}\right]$ and let $a_{i}=-a_{i i}$. We denote by $\pi=\left(\pi_{i}\right)$ the stationary distribution of $\{Z(t)\}$. Whenever the CTMC stays in state $i$, the fluid level of the buffer is increasing at rate $d_{i}$. $d_{i}$ is often

\footnotetext{
${ }^{1}$ This measure is refered to as first passage time in [3], but we follow a different naming convention because the analysis of the first passage time to a general fluid level is a more complicated problem than the analysis of the first passage time to empty buffer.
}

$\mathrm{RR} \mathrm{n}^{\circ} 4057$ 
refered to as the drift or the effective rate of state $i$. When $d_{i}<0$ it means that the fluid level is decreasing in the buffer. Of course, the fluid level can not decrease below $0 . Q(t)$ denotes the level of fluid in the buffer at time $t$. The dynamics of the fluid level process $\{Q(t), t \geq 0\}$ can be described as follows:

$$
\begin{array}{ll}
\frac{d Q(t)}{d t}=d_{Z(t)} \quad \text { when } \quad Q(t)>0 \\
\frac{d Q(t)}{d t}=\max \left(d_{Z(t)}, 0\right) \quad \text { when } \quad Q(t)=0
\end{array}
$$

\subsection{Analysis of busy period}

The busy period is the period of time while the buffer contains a positive amount of fluid (Figure 1). Without loss of generality we devote attention only to the first busy period in this paper. The length of further busy periods can be obtained as the special case when the initial fluid level is 0 . The random time $T$ is defined by

$$
T=\inf \{t>0 \mid Q(t)=0\}
$$

The distribution of the random time $T$ conditioned on the initial fluid level and on the initial state $Z(0)$ is defined as:

$$
F_{i}(t, x)=\operatorname{Pr}(T \leq t \mid Z(0)=i, Q(0)=x)
$$

By the given model definition $T=0$ if $Q(0)=0$ and $d_{i} \leq 0$, otherwise $\operatorname{Pr}(T=0)=0$.

Theorem $1 F_{i}(t, x)$ satisfies the backward differential equation

$$
\frac{\partial F_{i}(t, x)}{\partial t}-d_{i} \frac{\partial F_{i}(t, x)}{\partial x}=\sum_{k \in S} a_{i k} F_{k}(t, x) \quad \text { if } x>0
$$

with initial conditions

$$
\begin{aligned}
& F_{i}(t, 0)=1 \text { if } t \geq 0 \text { and } d_{i} \leq 0 \\
& F_{i}(0, x)=0 \text { if } x>0 \\
& F_{i}(0,0)=0 \text { if } d_{i}>0
\end{aligned}
$$




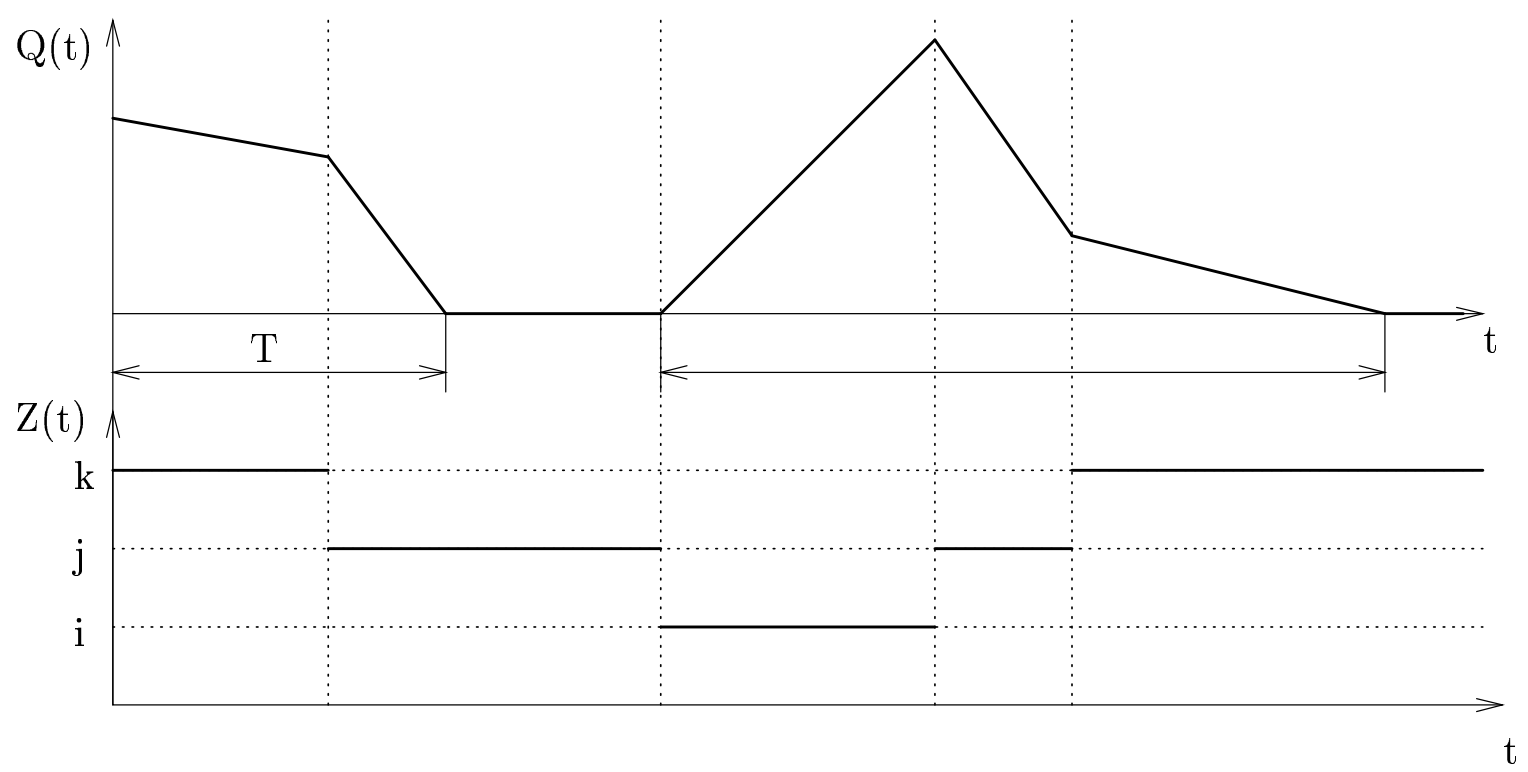

Figure 1: Busy periods of a stochastic fluid model

Proof. As mentioned in [3], the backward argument that describes the evolution of the process is:

$$
F_{i}(t, x)=\left(1-a_{i} \Delta\right) F_{i}\left(t-\Delta, x-d_{i} \Delta\right)+\sum_{k \in S, k \neq i} a_{i k} \Delta F_{k}\left(t-\Delta, x-d_{i} \Delta\right)+o(\Delta)
$$

which gives the theorem by algebraic manipulations and letting $\Delta \rightarrow 0$.

Let $|S|$ be the number of states in $S$ and let $m+1, m<|S|$, be the number of distinct values among all the effective rates $d_{i}$. These $m+1$ distinct effective rates are denoted by $r_{0}, r_{1}, \ldots, r_{m}$ and ordered as follows

$$
r_{m}>r_{m-1}>\ldots>r_{v} \geq 0>r_{v-1}>\ldots>r_{1}>r_{0}
$$

where $v$ is the number of negative effective rates. The state space $S$ of the process $X$ can then be divided into $m+1$ disjoint subsets $B_{m}, B_{m-1}, \ldots, B_{0}$ where $B_{i}$ is composed by the states $i$ of $S$ having the same effective rate $r_{i}$, that is $B_{i}=\left\{j \in S \mid d_{j}=r_{i}\right\} .\left|B_{i}\right|$ denotes the cardinality of subset $B_{i}$.

$\mathrm{RR} \mathrm{n}^{\circ} 4057$ 
If $v=0$ the buffer never becomes empty after time 0 , so we have $T=\infty$. Thus, we suppose without loss of generality that $v \geq 1$.

With this notation, we have, with probability 1 ,

$$
T \in \begin{cases}\bigcup_{j=0}^{v-1}\left[-\frac{x}{r_{j}},-\frac{x}{r_{j+1}^{+}}\right) & \text {if } x>0 \\ {[0, \infty)} & \text { if } x=0\end{cases}
$$

where $r_{j+1}^{+}=r_{j+1}$ for $j=0, \ldots, v-2$ and $r_{j+1}^{+}=0$ for $j=v-1$, so that $-x / r_{v}^{+}=+\infty$

For $x>0$, the distribution of $T$ has $v$ jumps at points $-x / r_{j}$ for $j=0, \ldots v-1$. If $x=0$, the distribution of $T$ has only one jump at point 0 . For $x>0$, the jump at point $-x / r_{j}$ corresponds to a sojourn of the Markov process $\{Z(t)\}$ in the subset $B_{j}$ that starts at time 0 and ends after time $-x / r_{j}$. These jumps are given, for $x>0$ and $j=0, \ldots, v-1$ by

$$
\operatorname{Pr}\left(T=-\frac{x}{r_{j}} \mid Z(0)=i, Q(0)=x\right)=\left\{\begin{array}{cl}
\left(e^{-A_{B_{j} B_{j}} \frac{x}{r_{j}}} \mathbb{1}_{B_{j}}\right)(i) & \text { if } i \in B_{j} \\
0 & \text { otherwise }
\end{array}\right.
$$

where $A_{B_{j} B_{j}}$ is the sub-infinitesimal generator of dimension $\left|B_{j}\right|$ obtained from $A$ by considering only the internal transitions of the subset $B_{j}$ and $\mathbb{1}_{B_{j}}$ is the column vector of dimension $\left|B_{j}\right|$ with all its entries equal to 1.

We denote by $P$ the transition probability matrix of the uniformized Markov chain associated to $\{Z(t)\}$ and by $\lambda$ the uniformization rate which verifies $\lambda \geq \max \left(a_{i}, i \in S\right)$. The matrix $P$ is then related to $A$ by $P=I+A / \lambda$, where $I$ denotes the identity matrix. In the following, to simplify notation, we will consider $\{Z(t)\}$ as the uniformized process. For every $i, j=0, \ldots, m$, we denote by $P_{B_{i} B_{j}}$ the submatrix of $P$ containing the transition probabilities from states of $B_{i}$ to states of $B_{j}$.

The distribution of the first time the buffer becomes empty, $T$, is given in the following theorem that applies the same approach as in [6]. The notation $0_{B_{l}}$ stands for the null column vector of dimension $\left|B_{l}\right|$.

Theorem 2 For every $i \in S$ and $x>0$, we have

$$
F_{i}(t, x)=\sum_{n=0}^{\infty} e^{-\lambda t} \frac{(\lambda t)^{n}}{n !} \sum_{k=0}^{n}\left(\begin{array}{l}
n \\
k
\end{array}\right) p_{j}^{k}\left(1-p_{j}\right)^{n-k} b_{i}^{(j)}(n, k)
$$


where $p_{j}=\frac{x+r_{j} t}{\left(r_{j}-r_{j+1}^{+}\right) t}$ if $t \in\left[-\frac{x}{r_{j}},-\frac{x}{r_{j+1}^{+}}\right)$, for $j=0,1, \ldots, v-1$. The coefficients $b_{i}^{(j)}(n, k)$ are given by the following recursive expressions on the column vectors $b_{B_{l}}^{(j)}(n, k)=$ $\left(b_{i}^{(j)}(n, k)\right)_{i \in B_{l}}$ for $0 \leq l \leq m$ and $0 \leq j \leq v-1$.

for $j+1 \leq l \leq m$ :

for $n \geq 0: b_{B_{l}}^{(0)}(n, 0)=0_{B_{l}}$ and $b_{B_{l}}^{(j)}(n, 0)=b_{B_{l}}^{(j-1)}(n, n)$ for $j>0$

for $1 \leq k \leq n: b_{B_{l}}^{(j)}(n, k)=\frac{r_{l}-r_{j+1}^{+}}{r_{l}-r_{j}} b_{B_{l}}^{(j)}(n, k-1)+\frac{r_{j+1}^{+}-r_{j}}{r_{l}-r_{j}} \sum_{i=0}^{m} P_{B_{l} B_{i}} b_{B_{i}}^{(j)}(n-1, k-1)$, for $0 \leq l \leq j$ :

for $n \geq 0: b_{B_{l}}^{(v-1)}(n, n)=\mathbb{1}_{B_{l}}$ and $b_{B_{l}}^{(j)}(n, n)=b_{B_{l}}^{(j+1)}(n, 0)$ for $j<v-1$

for $0 \leq k \leq n-1: b_{B_{l}}^{(j)}(n, k)=\frac{r_{j}-r_{l}}{r_{j+1}^{+}-r_{l}} b_{B_{l}}^{(j)}(n, k+1)+\frac{r_{j+1}^{+}-r_{j}}{r_{j+1}^{+}-r_{l}} \sum_{i=0}^{m} P_{B_{l} B_{i}} b_{B_{i}}^{(j)}(n-1, k)$.

Proof. See Appendix A.

The special case when the initial fluid level is 0 (i.e. $Q(0)=0$ ) is considered in the following corollary.

Corollary 3 For every $i \in S$, we have

$$
F_{i}(t, 0)=\sum_{n=0}^{\infty} e^{-\lambda t} \frac{(\lambda t)^{n}}{n !} b_{i}(n, n),
$$

where the coefficients $b_{i}(n, k)$ are given by the following recursive expressions on the column vectors $b_{B_{l}}(n, k)$ for $0 \leq l \leq m$

for $v \leq l \leq m$ :

for $n \geq 0: b_{B_{l}}(n, 0)=0_{B_{l}}$

for $1 \leq k \leq n: b_{B_{l}}(n, k)=\frac{r_{l}}{r_{l}-r_{v-1}} b_{B_{l}}(n, k-1)+\frac{-r_{v-1}}{r_{l}-r_{v-1}} \sum_{i=0}^{m} P_{B_{l} B_{i}} b_{B_{i}}(n-1, k-1)$,

for $0 \leq l \leq v-1$ :

$$
\begin{aligned}
& \text { for } n \geq 0: b_{B_{l}}(n, n)=\mathbb{1}_{B_{l}} \\
& \text { for } 0 \leq k \leq n-1: b_{B_{l}}(n, k)=\frac{r_{l}-r_{v-1}}{r_{l}} b_{B_{l}}(n, k+1)+\frac{r_{v-1}}{r_{l}} \sum_{i=0}^{m} P_{B_{l} B_{i}} b_{B_{i}}(n-1, k) .
\end{aligned}
$$


Proof. When $x=0$ we have $T \in[0,+\infty)$. This corresponds to the case $j=v-1$ in Theorem 2. By taking $x=0$ and $j=v-1$ in equation (5) we get relation (6) since in this case $p_{j}=1$. The recurrence relation satisfied by the $b_{B_{l}}(n, k)$ are then easily obtained by taking $j=v-1$ in the recurrence relation of Theorem 2 .

Note that the relation (7) and (8) are convex combinations of vectors since we have

$$
0 \leq \frac{r_{l}}{r_{l}-r_{v-1}}=1-\frac{-r_{v-1}}{r_{l}-r_{v-1}} \leq 1, \text { for } v \leq l \leq m
$$

and

$$
0 \leq \frac{r_{l}-r_{v-1}}{r_{l}}=1-\frac{r_{v-1}}{r_{l}} \leq 1 \text { for } 0 \leq l \leq v-1
$$

\subsection{Computational properties of the numerical procedure}

In practical applications the analysis of busy period with initially empty buffer is much more common. Fortunately, both the computational complexity and the memory requirement of the numerical method based on Corollary 3 is $v$ (the number of negative distinct drift values) times less in this case. The computational complexity of the analysis procedure can be further reduced using the results provided in the following theorem.

Theorem 4 The $b_{B_{l}}(n, k)$ vectors piecewise satisfy the following inequalities:

a) $0_{B_{l}} \leq b_{B_{l}}(n, k) \leq \mathbb{1}_{B_{l}}$ for $0 \leq l \leq m, n \geq 0,0 \leq k \leq n$

b) $b_{B_{l}}(n, k) \leq b_{B_{l}}(n+1, k+1)$ for $0 \leq l \leq m, n \geq 0,0 \leq k \leq n$,

c) $b_{B_{l}}(n, k) \geq b_{B_{l}}(n+1, k)$ for $0 \leq l \leq m, n \geq 0,0 \leq k \leq n$

d) $b_{B_{l}}(n, k) \leq b_{B_{l}}(n, k+1)$ for $0 \leq l \leq m, n \geq 0,0 \leq k<n$,

e) $b_{B_{l}}(n, k) \geq b_{B_{l}}(n+1, k-1) \quad$ for $0 \leq l \leq m, n \geq 0,1 \leq k \leq n$,

f) $\lim _{n \rightarrow \infty} b_{B_{l}}(n, n)=\mathbb{1}_{B_{l}}$ for $0 \leq l \leq m$.

Proof. See Appendix B. 


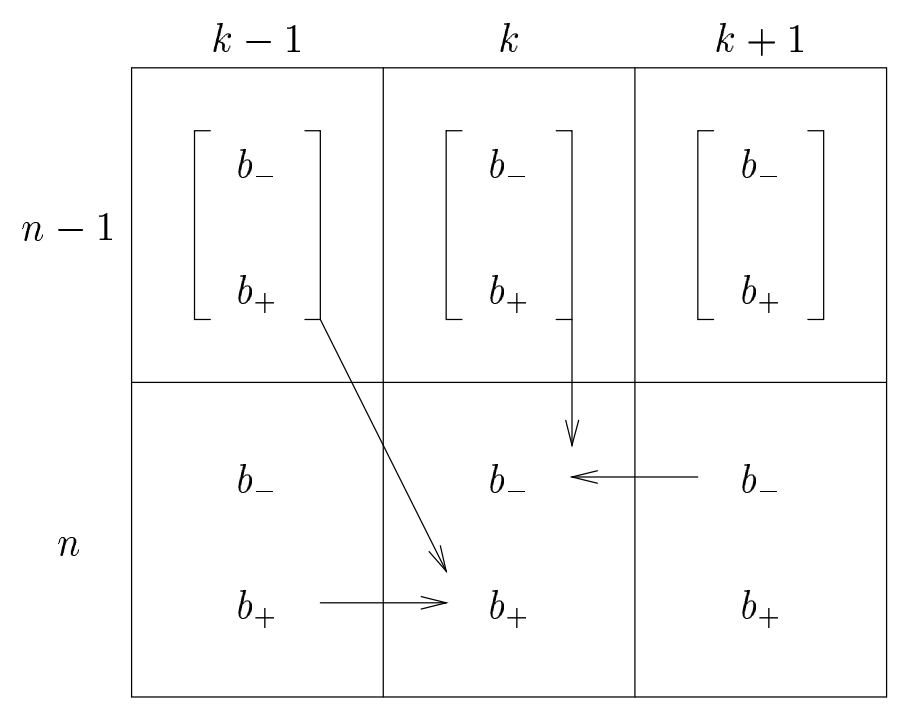

Figure 2: Computation of the $b_{i}(n, k)$

The computation of the $b_{i}(n, k)$ can be illustrated using figure 2 . In this figure, we represent the $b_{i}(n, k)$ using column vectors $b_{-}(n, k)=\left(b_{B_{l}}(n, k)\right)_{l=0, \ldots, v-1}$ and $b_{+}(n, k)=$ $\left(b_{B_{l}}(n, k)\right)_{l=v, \ldots, m}$ and we show graphically the relation (7) and (8) used for the computation of $b_{+}(n, k)$ and $b_{-}(n, k)$.

We assume that the stability condition, $\sum_{j \in S} d_{j} \pi_{j}<0$, is satisfied, so that the random time $T$ is finite a.s. Suppose, without any loss of generality, that the initial state $i$ is fixed. For a given error tolerance $\varepsilon$, we define integer $N^{\prime}$ as

$$
N^{\prime}=\min \left\{n \in \mathbb{N} \mid\left(1-b_{i}(n, n)\right)\left(1-\sum_{r=0}^{n} e^{-\lambda t} \frac{(\lambda t)^{r}}{r !}\right) \leq \varepsilon\right\} .
$$

Note that the value of $N^{\prime}$ will be known only a posteriori since it depends on the $b_{i}(n, k)$. An upper bound of $N$, available a priori, that is before the computation of the $b_{i}(n, k)$, is the classical truncation step of the Poisson series given by

$$
N=\min \left\{n \in \mathbb{N} \mid\left(1-\sum_{r=0}^{n} e^{-\lambda t} \frac{(\lambda t)^{r}}{r !}\right) \leq \varepsilon\right\} \text {. }
$$

$\mathrm{RR} \mathrm{n}^{\circ} 4057$ 
From Theorem 4, inequality a), we obtain $N^{\prime} \leq N$. Using the truncation step $N^{\prime}$, we get

$$
F_{i}(t, 0)=1-\sum_{n=0}^{N^{\prime}} e^{-\lambda t} \frac{(\lambda t)^{n}}{n !}+\sum_{n=0}^{N^{\prime}} e^{-\lambda t} \frac{(\lambda t)^{n}}{n !} b_{i}(n, n)-e\left(N^{\prime}\right)
$$

where the rest of the series $e\left(N^{\prime}\right)$ satisfies

$$
e\left(N^{\prime}\right)=\sum_{n=N^{\prime}+1}^{\infty} e^{-\lambda t} \frac{(\lambda t)^{n}}{n !}\left(1-b_{i}(n, n)\right) \leq\left(1-b_{i}\left(N^{\prime}, N^{\prime}\right)\right)\left(1-\sum_{n=0}^{N^{\prime}} e^{-\lambda t} \frac{(\lambda t)^{n}}{n !}\right) \leq \varepsilon
$$

Another way to reduce the computational complexity is to avoid the calculation of the vectors $b_{B_{l}}(n, k)$ when all of their components are less than or equal to a given value $\varepsilon^{\prime}$. It is easy to check based on expression (7) that if the vectors $b_{B_{l}}(n, k-1)$ and $b_{B_{i}}(n-$ $1, k-1)$ have all their entries less than or equal to $\varepsilon^{\prime}$ then the vector $b_{B_{l}}(n, k)$ has also all its entries less than or equal to $\varepsilon^{\prime}$. The same result holds for the appropriate terms in expression (8). This property is due to the fact that both expressions (7) and (8) are convex combinations of vectors. This property together with Theorem 4 suggest us to further reduce the computation of $b_{B_{l}}(n, k)$ vectors. More precisely, let us define, for a given value of $\varepsilon^{\prime}$ the integers $N_{0}, N_{1}, \ldots$ and $N^{\prime \prime}$ as

$$
N_{0}=\min \left\{1 \leq n \leq N^{\prime \prime}-1 \mid b_{B_{l}}(n, 0) \leq \varepsilon^{\prime} \mathbb{1}_{B_{l}} \text { for } l=0, \ldots, v-1\right\},
$$

for $h \geq 1$,

$$
N_{h}=\min \left\{N_{h-1}+1 \leq n \leq N^{\prime \prime}-1 \mid b_{B_{l}}^{[h]}(n, h) \leq \varepsilon^{\prime} \mathbb{1}_{B_{l}} \text { for } l=0, \ldots, v-1\right\}
$$

and,

$$
N^{\prime \prime}=\min \left\{n \in \mathbb{N} \mid\left(1-b_{i}^{[H+1]}(n, n)\right)\left(1-\sum_{r=0}^{n} e^{-\lambda t} \frac{(\lambda t)^{r}}{r !}\right) \leq \varepsilon\right\}
$$

where,

1) for $0 \leq l \leq m, 0 \leq n \leq N_{0}$, and $0 \leq k \leq n$ :

$$
b_{B_{l}}^{[0]}(n, k)=b_{B_{l}}(n, k)
$$


2) for $h \geq 1,0 \leq l \leq m$ and $h \leq k \leq N_{h-1}$ :

$$
b_{B_{l}}^{[h]}\left(N_{h-1}, k\right)=b_{B_{l}}^{[h-1]}\left(N_{h-1}, k\right),
$$

3) for $h \geq 1$ and $0 \leq l \leq m$ :

for $v \leq l \leq m, N_{h-1}+1 \leq n$ and $h+1 \leq k \leq n$ :

$$
\begin{aligned}
& b_{B_{l}}^{[h]}(n, h)=0_{B_{l}} \\
& b_{B_{l}}^{[h]}(n, k)=\frac{r_{l}}{r_{l}-r_{v-1}} b_{B_{l}}^{[h]}(n, k-1)+\frac{-r_{v-1}}{r_{l}-r_{v-1}} \sum_{i=0}^{m} P_{B_{l} B_{i}} b_{B_{i}}^{[h]}(n-1, k-1),
\end{aligned}
$$

for $0 \leq l \leq v-1, N_{h-1}+1 \leq n$ and $h \leq k \leq n-1$ :

$$
\begin{aligned}
& b_{B_{l}}^{[h]}(n, n)=\mathbb{1}_{B_{l}} \\
& b_{B_{l}}^{[h]}(n, k)=\frac{r_{l}-r_{v-1}}{r_{l}} b_{B_{l}}^{[h]}(n, k+1)+\frac{r_{v-1}}{r_{l}} \sum_{i=0}^{m} P_{B_{l} B_{i}} b_{B_{i}}^{[h]}(n-1, k),
\end{aligned}
$$

4) the index of the greatest considered $N_{h}$ is

$$
H=\max \left\{h \mid N_{h} \leq N^{\prime \prime}-1\right\} .
$$

In the above list, Item 1) represents the initialization step for $h=0$, and Item 2) for $h \geq 1$. Item 3) provides the application of (7) and (8) and the approximation of the negligible vectors. Finally, Item 4 ) defines the greatest level of reduction used in the numerical method. Note that $N^{\prime \prime}$ as well as $N_{h}(0 \leq h \leq H)$ and $H$ are obtained during the execution of the numerical procedure (a posteriori). By the definition of $N_{h}$, we have $h+1 \leq N_{h} \leq N^{\prime \prime}-1$, so $H \leq N^{\prime \prime}-2$.

All these mechanisms are illustrated in figure 3 , where $H=3$. In this figure, we represent the initial conditions for vectors $b_{-}(n, k)$ and $b_{+}(n, k)$ described in figure 2 . The vector $\mathbb{1}$ means that we have $b_{-}^{[h]}(n, n)=\mathbb{1}_{-}$and $\varepsilon^{\prime}$ means that $b_{-}^{[h]}\left(N_{h}, h\right) \leq \varepsilon^{\prime} \mathbb{1}_{-}$by definition of $N_{h}$. We obtain, in particular, from relation $(7)$, that $b_{+}^{[h+1]}\left(N_{h}+1, h+1\right) \leq \varepsilon^{\prime} \mathbb{1}_{+}$and so, to avoid its computation we set $b_{+}^{[h+1]}\left(N_{h}+1, h+1\right)=0_{+}$and we also set $b_{+}^{[h+1]}(n, h+1)=0_{+}$ for $n \geq N_{h}+2$. The cells in gray, in figure 3 , are not calculated.

Let us now evaluate the error introduced by the use of the $b_{B_{l}}^{[h]}(n, k)$ instead of the $b_{B_{l}}(n, k)$. It is easy to check that Theorem 4 is still valid for all the $b_{B_{l}}^{[h]}(n, k), h=0, \ldots, H+1$. 


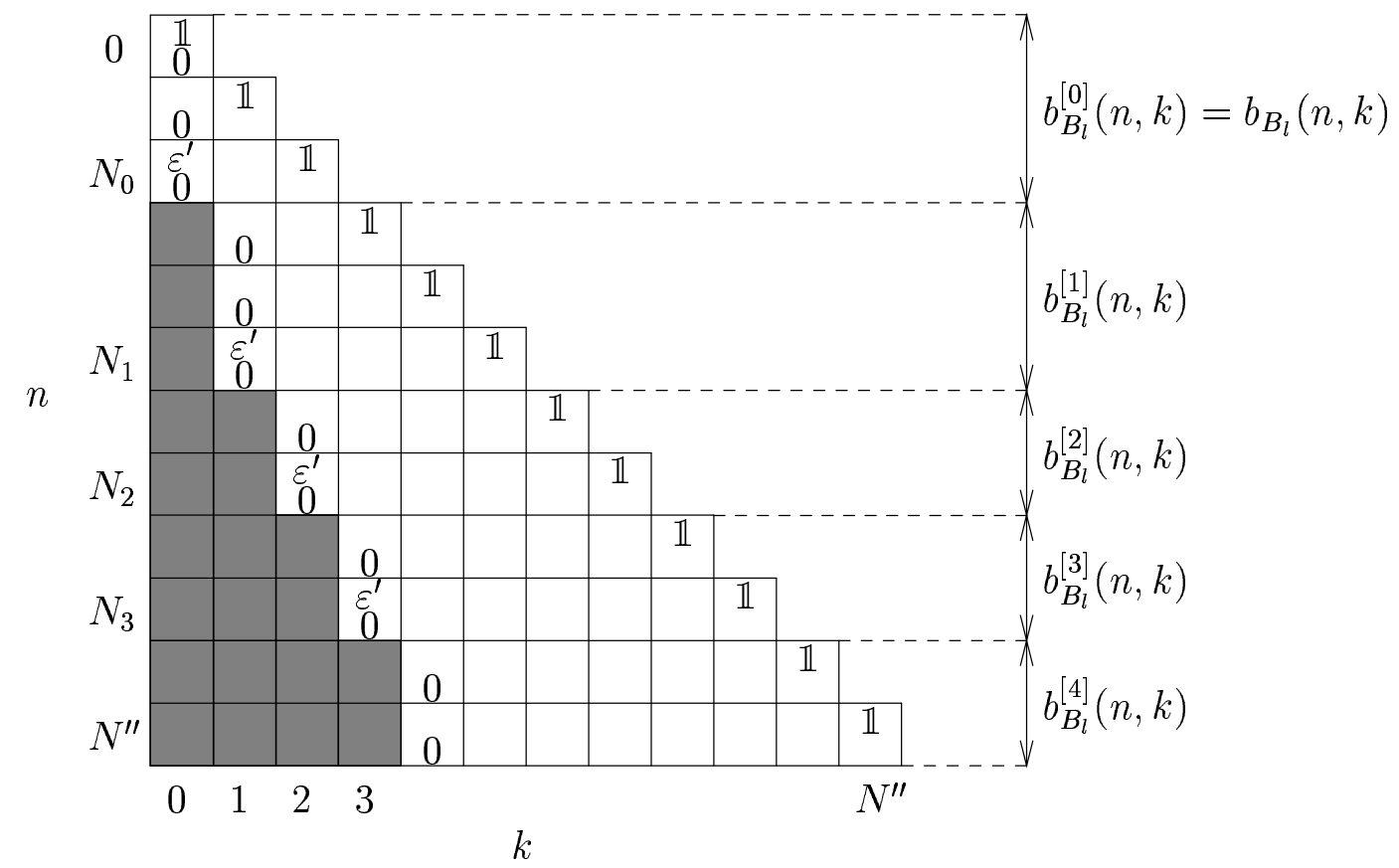

Figure 3: In gray, the cells $(n, k)$ that are not computed 
It follows in particular that the integer $N^{\prime \prime}$ exists. Moreover, for $h \geq 1, n \geq N_{h-1}+1, h \leq$ $k \leq n$, and $l=0, \ldots, m$, we have $b_{B_{l}}^{[h]}(n, k) \leq b_{B_{l}}^{[h-1]}(n, k)$ since we start the computation of the $b_{B_{l}}^{[h]}(n, k)$ when, for $l=0, \ldots, v-1, b_{B_{l}}^{[h-1]}\left(N_{h-1}, h-1\right) \leq \varepsilon^{\prime}$ and we set $b_{B_{l}}^{[h]}\left(N_{h-1}+1, h\right)=0$, for $l=v, \ldots, m$. based on these remarks, it can be easily checked by induction that for every $i \in S, n \geq 0,0 \leq k \leq n$, and $h=1, \ldots, H+1$, we have

$$
0 \leq b_{i}^{[h-1]}(n, k)-b_{i}^{[h]}(n, k) \leq \varepsilon^{\prime} .
$$

In order to simplify writing, we define $N_{-1}=-1$ and $N_{H+1}=N^{\prime \prime}$. The quantity that is really computed is $\widetilde{F}_{i}(t)$ which is given by

$$
\widetilde{F}_{i}(t)=1-\sum_{n=0}^{N^{\prime \prime}} e^{-\lambda t} \frac{(\lambda t)^{n}}{n !}+\sum_{h=0}^{H+1} \sum_{n=N_{h-1}+1}^{N_{h}} e^{-\lambda t} \frac{(\lambda t)^{n}}{n !} b_{i}^{[h]}(n, n) .
$$

Let us denote by $E$ the error so obtained. We have

$$
\begin{aligned}
E & =F_{i}(t, 0)-\widetilde{F}_{i}(t) \\
& =\sum_{n=N^{\prime \prime}+1}^{\infty} e^{-\lambda t} \frac{(\lambda t)^{n}}{n !}\left(1-b_{i}(n, n)\right)-\sum_{h=0}^{H+1} \sum_{n=N_{h-1}+1}^{N_{h}} e^{-\lambda t} \frac{(\lambda t)^{n}}{n !}\left(b_{i}(n, n)-b_{i}^{[h]}(n, n)\right) .
\end{aligned}
$$

We denote respectively by $e_{1}\left(N^{\prime \prime}\right)$ and $e_{2}\left(N^{\prime \prime}\right)$ the first and second term of the right hand side. From Theorem 4, and (13) and (12), we have that

$$
\begin{aligned}
0 \leq e_{1}\left(N^{\prime \prime}\right) & =\sum_{n=N^{\prime \prime}+1}^{\infty} e^{-\lambda t} \frac{(\lambda t)^{n}}{n !}\left(1-b_{i}(n, n)\right) \leq\left(1-b_{i}\left(N^{\prime \prime}, N^{\prime \prime}\right)\right)\left(1-\sum_{n=0}^{N^{\prime \prime}} e^{-\lambda t} \frac{(\lambda t)^{n}}{n !}\right) \\
& \leq\left(1-b_{i}^{[H+1]}\left(N^{\prime \prime}, N^{\prime \prime}\right)\right)\left(1-\sum_{n=0}^{N^{\prime \prime}} e^{-\lambda t} \frac{(\lambda t)^{n}}{n !}\right) \\
& \leq \varepsilon .
\end{aligned}
$$

For $h=0$, we have by definition $b_{i}(n, n)=b_{i}^{[0]}(n, n)$, and for $h \geq 1$, we have

$$
0 \leq b_{i}(n, n)-b_{i}^{[h]}(n, n)=\sum_{u=1}^{h}\left(b_{i}^{[u-1]}(n, n)-b_{i}^{[u]}(n, n)\right) \leq h \varepsilon^{\prime} .
$$

$\mathrm{RR} \mathrm{n}^{\circ} 4057$ 
Thus, we get from inequality (13),

$$
\begin{aligned}
0 \leq e_{2}\left(N^{\prime \prime}\right) & =\sum_{h=1}^{H+1} \sum_{n=N_{h-1}+1}^{N_{h}} e^{-\lambda t} \frac{(\lambda t)^{n}}{n !}\left(b_{i}(n, n)-b_{i}^{[h]}(n, n)\right) \\
& \leq \varepsilon^{\prime} \sum_{h=1}^{H+1} h \sum_{n=N_{h-1}+1}^{N_{h}} e^{-\lambda t} \frac{(\lambda t)^{n}}{n !} \\
& \leq(H+1) \varepsilon^{\prime} \\
& \leq(N-1) \varepsilon^{\prime} .
\end{aligned}
$$

By choosing $\varepsilon^{\prime}=\varepsilon /(N-1)$, where $N$ is known a priori, we get $0 \leq e_{1}\left(N^{\prime \prime}\right) \leq \varepsilon$ and $0 \leq e_{2}\left(N^{\prime \prime}\right) \leq \varepsilon$ so $|E|=\left|e_{1}\left(N^{\prime \prime}\right)-e_{2}\left(N^{\prime \prime}\right)\right| \leq \varepsilon$.

The pseudocode of the algorithm is given in Table 1 . In this algorithm, the $b_{B_{l}}^{[h]}(n, k)$ are computed successively for the different values of $h$ and are all stored in the $b_{B_{l}}(n, k)$ according to figure 3 and thanks to Item 2).

Remark : The truncation levels $N, N^{\prime}$ and $N^{\prime \prime}$ are in fact functions of $t$. In order to compute $F_{i}(t, 0)$ for several values of $t$, say $t_{1}<\cdots<t_{M}$, we only need to determine these truncation levels for the highest value $t_{M}$ since the rest of the Poisson series, which is used to bound the errors, is an increasing function of $t$. 
input : $\varepsilon, i, t_{1}<\cdots<t_{M}$.

output : $\widetilde{F}_{i}\left(t_{j}\right)$, for $j=1, \ldots, M$.

Compute $N$ from relation (10) with $t=t_{M}$;

$N^{\prime \prime}=N$;

$\varepsilon^{\prime}=\varepsilon /(N-1)$

for $l=0$ to $v-1$ do $b_{B_{l}}(0,0)=\mathbb{1}_{B_{l}}$; endfor

for $l=v$ to $m$ do $b_{B_{l}}(0,0)=0_{B_{l}}$; endfor

$\mathrm{h}=0$;

for $n=1$ to $N$ do

for $l=0$ to $v-1$ do $b_{B_{l}}(n, n)=\mathbb{1}_{B_{l}} ;$ endfor

for $k=n-1$ downto $h$ do

for $l=0$ to $v-1$ do compute $b_{B_{l}}(n, k)$ from relation (8); endfor endfor

for $l=v$ to $m$ do $b_{B_{l}}(n, h)=0_{B_{l}}$; endfor

for $k=h+1$ to $n$ do

for $l=v$ to $m$ do compute $b_{B_{l}}(n, k)$ from relation (7); endfor endfor

if $\left(b_{B_{l}}(n, h) \leq \varepsilon^{\prime} \mathbb{1}_{B_{l}}, \forall l=0, \ldots, v-1\right)$ then $N_{h}=n ; h=h+1$; endif

if $\left(\left(1-b_{i}(n, n)\right)\left(1-\sum_{r=0}^{n} e^{-\lambda t_{M}} \frac{\left(\lambda t_{M}\right)^{r}}{r !}\right) \leq \varepsilon\right)$ then $N^{\prime \prime}=n$; break; endif

endfor

for $j=1$ to $M$ do $\widetilde{F}_{i}\left(t_{j}\right)=1-\sum_{n=0}^{N^{\prime \prime}} e^{-\lambda t_{j}} \frac{\left(\lambda t_{j}\right)^{n}}{n !}+\sum_{n=0}^{N^{\prime \prime}} e^{-\lambda t_{j}} \frac{\left(\lambda t_{j}\right)^{n}}{n !} b_{i}(n, n)$;

Table 1: Algorithm for the computation of the busy period distribution

\section{Numerical example}

The distribution of the busy period and its dependence on the initial state of the busy period are analyzed in this section. The considered fluid process is generated by $m$ identical on-off sources whose on and off periods are exponentially distributed with parameter $\beta$ and $\gamma$, respectively. The sources generate fluid at rate $\theta$ during their on period, and do not generate any fluid during their off period. The fluid generated by the sources is driven to an

$\mathrm{RR} \mathrm{n}^{\circ} 4057$ 
infinite buffer whose exit rate is $c$. In this case the Markov chain that determines the fluid accumulation has $m+1$ states. Assuming the states are numbered from 0 to $m$ according to the number of on sources $(Z(t)=$ \#on sources) the drift of state $i$ is $i \theta-c$. Since the busy ratio of a source is $\gamma /(\gamma+\beta)$ the utilization of the fluid system is

$$
\rho=\frac{\theta m \gamma}{c(\gamma+\beta)}
$$

Figure 4 depicts the distribution of the busy period of the fluid system with the following set of parameters: $m=40, \beta=1, \gamma=0.2, \theta=1, c=7.8(\rightarrow \rho=0.854701), Q(0)=0$, and $\varepsilon=10^{-5}$. The solid line represents the case when the initial state at the beginning of the busy period is the one with minimal sources of on sources (i.e., $Z(0)=\min \{i \mid i \theta-c>0\}$ ), which is $Z(0)=8$ in this case, while the dotted line represents the case when all the sources are in the on state at the beginning of the busy period, i.e. $Z(0)=m$.

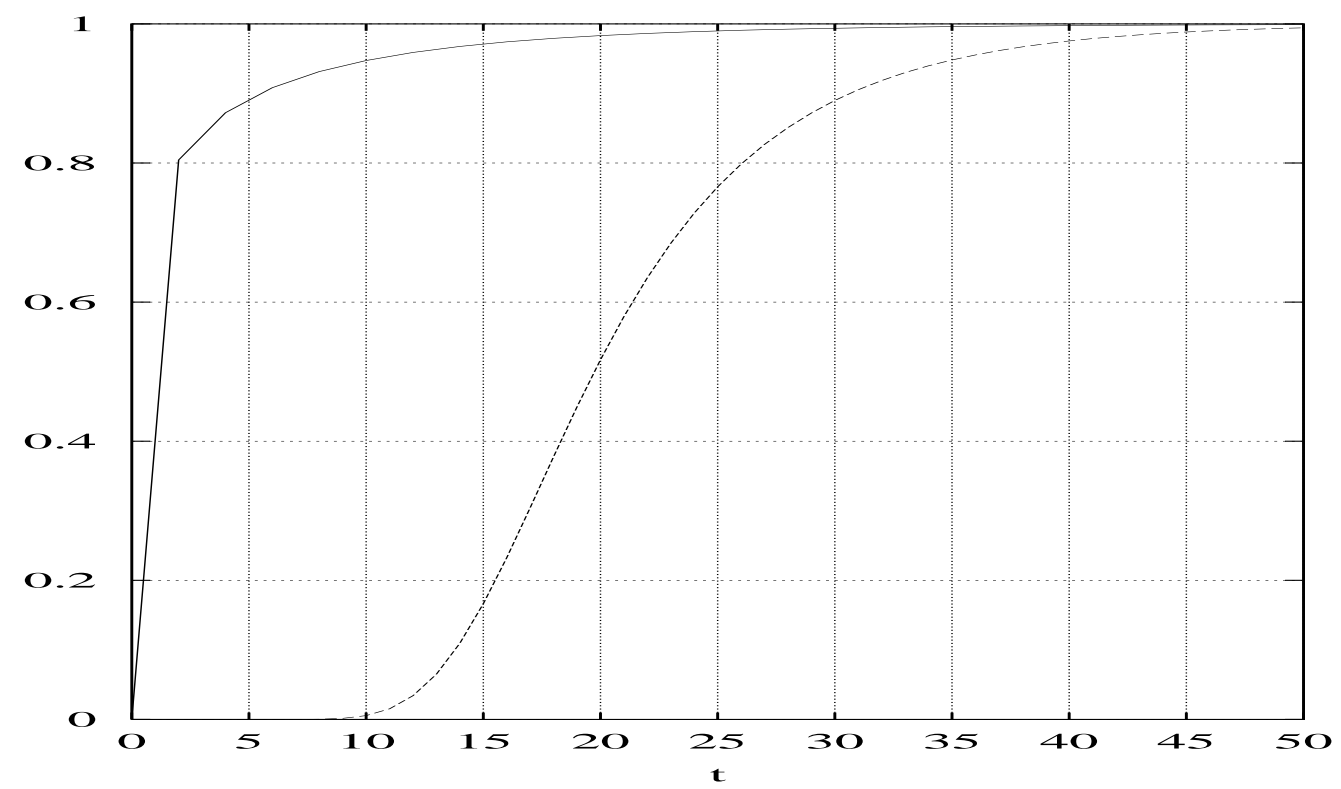

Figure 4: Distribution of the busy period with different initial states

To evaluate the benefit of the numerical procedure based on Theorem 4 the same fluid model with identical on-off sources was evaluated with a different set of parameters: $m=4$, $\beta=1, \gamma=0.2, \theta=1, c=0.8(\rightarrow \rho=0.833333), Q(0)=0, Z(0)=m$, and $\varepsilon=10^{-5}$. The 
obtained uniformization rate is $\lambda=4$ and the maximal time at which the distribution is evaluated is $t=100$ (i.e., $\lambda t=400$ ).

With these parameters the truncation of the randomization method with respect to $\varepsilon$ is at $N=488$ and the value of $N^{\prime}$ truncation was obtained at 470 .

The computational cost of an iteration cycle reduced significantly when the procedure using the truncation steps $N_{h}$ is used. With this procedure, we got $N^{\prime \prime}=470, H=160$. Some of the values of the $N_{h}$ are : $N_{0}=80, N_{1}=88, N_{50}=243, N_{100}=352, N_{150}=450$ and the last one is $N_{160}=469$. The number of cells whose calculation has been avoided is equal to $\sum_{h=0}^{H}\left(N-N_{h}\right)=27512$. This number represents approximatively $25 \%$ of the total number of cells, which is $\left(N^{\prime \prime}+1\right)\left(N^{\prime \prime}+2\right) / 2=111156$.

\section{Appendix A. Proof of Theorem 2}

For $x>0$ and $t \in\left(-\frac{x}{r_{j}},-\frac{x}{r_{j+1}^{+}}\right)$for $j=0,1, \ldots, v-1$, we write the solution of equation (3) for every $i \in S$, as

$$
F_{i}(t, x)=\sum_{n=0}^{\infty} e^{-\lambda t} \frac{(\lambda t)^{n}}{n !} \sum_{k=0}^{n}\left(\begin{array}{l}
n \\
k
\end{array}\right) p_{j}^{k}\left(1-p_{j}\right)^{n-k} b_{i}^{(j)}(n, k),
$$

and we determine the relations that must be satisfied by the coefficients $b_{i}^{(j)}(n, k)$. We then have

$$
\begin{aligned}
& \frac{\partial F_{i}(t, x)}{\partial t}=-\lambda F_{i}(t, x) \\
&+e^{-\lambda t} \frac{\partial}{\partial t}\left[\sum_{n=1}^{\infty} \frac{\left(\frac{\lambda}{r_{j}-r_{j+1}^{+}}\right)^{n}}{n !} \sum_{k=0}^{n}\left(\begin{array}{c}
n \\
k
\end{array}\right)\left(x+r_{j} t\right)^{k}\left(-x-r_{j+1}^{+} t\right)^{n-k} b_{i}^{(j)}(n, k)\right] \\
&=-\lambda F_{i}(t, x)+e^{-\lambda t}\left[\sum_{n=1}^{\infty} \frac{\left(\frac{\lambda}{r_{j}-r_{j+1}^{+}}\right)^{n}}{n !} \sum_{k=1}^{n} k\left(\begin{array}{c}
n \\
k
\end{array}\right)\left(x+r_{j} t\right)^{k-1}\left(-x-r_{j+1}^{+} t\right)^{n-k} r_{j} b_{i}^{(j)}(n, k)\right. \\
&\left.\quad-\sum_{n=1}^{\infty} \frac{\left(\frac{\lambda}{r_{j}-r_{j+1}^{+}}\right)^{n}}{n !} \sum_{k=0}^{n-1}(n-k)\left(\begin{array}{c}
n \\
k
\end{array}\right)\left(x+r_{j} t\right)^{k}\left(-x-r_{j+1}^{+} t\right)^{n-k-1} r_{j+1}^{+} b_{i}^{(j)}(n, k)\right]
\end{aligned}
$$

$\mathrm{RR} \quad \mathrm{n}^{\circ} 4057$ 


$$
\begin{aligned}
= & -\lambda F_{i}(t, x) \\
& +e^{-\lambda t}\left[\sum_{n=1}^{\infty} \frac{\left(\frac{\lambda}{r_{j}-r_{j+1}^{+}}\right)^{n}}{n !} \sum_{k=0}^{n-1}(k+1)\left(\begin{array}{c}
n \\
k+1
\end{array}\right)\left(x+r_{j} t\right)^{k}\left(-x-r_{j+1}^{+} t\right)^{n-k-1} r_{j} b_{i}^{(j)}(n, k+1)\right. \\
=-\lambda F_{i}(t, x) & \left.-\sum_{n=1}^{\infty} \frac{\left(\frac{\lambda}{r_{j}-r_{j+1}^{+}}\right)^{n}}{n !} \sum_{k=0}^{n-1}(n-k)\left(\begin{array}{c}
n \\
k
\end{array}\right)\left(x+r_{j} t\right)^{k}\left(-x-r_{j+1}^{+} t\right)^{n-k-1} r_{j+1}^{+} b_{i}^{(j)}(n, k)\right] \\
+ & e^{-\lambda t}\left[\sum_{n=0}^{\infty} \frac{\left(\frac{\lambda}{r_{j}-r_{j+1}^{+}}\right)^{n+1}}{(n+1) !} \sum_{k=0}^{n}(k+1)\left(\begin{array}{c}
n+1 \\
k+1
\end{array}\right)\left(x+r_{j} t\right)^{k}\left(-x-r_{j+1}^{+} t\right)^{n-k} r_{j} b_{i}^{(j)}(n+1, k+1)\right. \\
& \left.-\sum_{n=0}^{\infty} \frac{\left(\frac{\lambda}{r_{j}-r_{j+1}^{+}}\right)^{n+1}}{(n+1) !} \sum_{k=0}^{n}(n-k+1)\left(\begin{array}{c}
n+1 \\
k
\end{array}\right)\left(x+r_{j} t\right)^{k}\left(-x-r_{j+1}^{+} t\right)^{n-k} r_{j+1}^{+} b_{i}^{(j)}(n+1, k)\right]
\end{aligned}
$$

For every $n \geq 0$ and $0 \leq k \leq n$ we have

$$
\frac{k+1}{n+1}\left(\begin{array}{c}
n+1 \\
k+1
\end{array}\right)=\frac{n-k+1}{n+1}\left(\begin{array}{c}
n+1 \\
k
\end{array}\right)=\left(\begin{array}{l}
n \\
k
\end{array}\right),
$$

so we obtain

$$
\begin{aligned}
& \frac{\partial F_{i}(t, x)}{\partial t}=-\lambda F_{i}(t, x) \\
& \quad+\frac{\lambda}{r_{j}-r_{j+1}^{+}} \sum_{n=0}^{\infty} e^{-\lambda t} \frac{(\lambda t)^{n}}{n !} \sum_{k=0}^{n}\left(\begin{array}{c}
n \\
k
\end{array}\right) p_{j}^{k}\left(1-p_{j}\right)^{n-k}\left[r_{j} b_{i}^{(j)}(n+1, k+1)-r_{j+1}^{+} b_{i}^{(j)}(n+1, k)\right] .
\end{aligned}
$$

In the same way, we have

$$
\frac{\partial F_{i}(t, x)}{\partial x}=\frac{\lambda}{r_{j}-r_{j+1}^{+}} \sum_{n=0}^{\infty} e^{-\lambda t} \frac{(\lambda t)^{n}}{n !} \sum_{k=0}^{n}\left(\begin{array}{c}
n \\
k
\end{array}\right) p_{j}^{k}\left(1-p_{j}\right)^{n-k}\left[b_{i}^{(j)}(n+1, k+1)-b_{i}^{(j)}(n+1, k)\right] .
$$


Using the uniformization technique, we have

$$
\sum_{r \in S} a_{i r} F_{r}(t, x)=-\lambda F_{i}(t, x)+\lambda \sum_{r \in S} p_{i r} F_{r}(t, x)
$$

that is,

$$
\sum_{r \in S} a_{i r} F_{r}(t, x)=-\lambda F_{i}(t, x)+\lambda \sum_{n=0}^{\infty} e^{-\lambda t} \frac{(\lambda t)^{n}}{n !} \sum_{k=0}^{n}\left(\begin{array}{l}
n \\
k
\end{array}\right) p_{j}^{k}\left(1-p_{j}\right)^{n-k} \sum_{r \in S} p_{i r} b_{r}^{(j)}(n, k) .
$$

It follows that if the $b_{i}^{(j)}(n, k)$ are such that

$$
\left(r_{j}-d_{i}\right) b_{i}^{(j)}(n+1, k+1)+\left(d_{i}-r_{j+1}^{+}\right) b_{i}^{(j)}(n+1, k)=\left(r_{j}-r_{j+1}^{+}\right) \sum_{r \in S} p_{i r} b_{r}^{(j)}(n, k)
$$

then equation (3) is satisfied.

The recurrence relation (15) can also be written as follows, for $j=0, \ldots, v-1$.

For $i \in B_{j+1} \cup \cdots \cup B_{m}$,

$$
b_{i}^{(j)}(n, k)=\frac{d_{i}-r_{j+1}^{+}}{d_{i}-r_{j}} b_{i}^{(j)}(n, k-1)+\frac{r_{j+1}^{+}-r_{j}}{d_{i}-r_{j}} \sum_{r \in S} p_{i r} b_{r}^{(j)}(n-1, k-1)
$$

and for $i \in B_{0} \cup \cdots \cup B_{j}$,

$$
b_{i}^{(j)}(n, k)=\frac{r_{j}-d_{i}}{r_{j+1}^{+}-d_{i}} b_{i}^{(j)}(n, k+1)+\frac{r_{j+1}^{+}-r_{j}}{r_{j+1}^{+}-d_{i}} \sum_{r \in S} p_{i r} b_{r}^{(j)}(n-1, k) .
$$

Using matrix and vector notation, we get for $j=0, \ldots, v-1$ and

for $j+1 \leq l \leq m$

$$
b_{B_{l}}^{(j)}(n, k)=\frac{r_{l}-r_{j+1}^{+}}{r_{l}-r_{j}} b_{B_{l}}^{(j)}(n, k-1)+\frac{r_{j+1}^{+}-r_{j}}{r_{l}-r_{j}} \sum_{i=0}^{m} P_{B_{l} B_{i}} b_{B_{i}}^{(j)}(n-1, k-1),
$$

for $0 \leq l \leq j$

$$
b_{B_{l}}^{(j)}(n, k)=\frac{r_{j}-r_{l}}{r_{j+1}^{+}-r_{l}} b_{B_{l}}^{(j)}(n, k+1)+\frac{r_{j+1}^{+}-r_{j}}{r_{j+1}^{+}-r_{l}} \sum_{i=0}^{m} P_{B_{l} B_{i}} b_{B_{i}}^{(j)}(n-1, k) .
$$

To get the initial conditions for the $b_{i}^{(j)}(n, k)$, we consider the jumps of $F_{i}(t, x)$ given by relation (4) in which we write

$$
e^{-A_{B_{j} B_{j}} \frac{x}{r_{j}}}=\sum_{n=0}^{\infty} e^{\lambda \frac{x}{r_{j}}} \frac{\left(-\lambda \frac{x}{r_{j}}\right)^{n}}{n !} P_{B_{j} B_{j}}^{n} .
$$

$\mathrm{RR} \quad \mathrm{n}^{\circ} 4057$ 
For every $j=0,1, \ldots, v-1$ we have $p_{j}=0$ when $t=-x / r_{j}$ and $p_{j} \longrightarrow 1$ when $t \longrightarrow-x / r_{j+1}^{+}, t<-x / r_{j+1}^{+}$, and so

$$
F_{i}\left(-\frac{x}{r_{j}}, x\right)=\sum_{n=0}^{\infty} e^{\lambda \frac{x}{r_{j}}} \frac{\left(-\lambda \frac{x}{r_{j}}\right)^{n}}{n !} b_{i}^{(j)}(n, 0)
$$

and, for $j<v-1$,

$$
\lim _{t \rightarrow \frac{-x}{r_{j+1}}, t<\frac{-x}{r_{j+1}}} F_{i}(t, x)=\sum_{n=0}^{\infty} e^{\lambda \frac{x}{r_{j+1}}} \frac{\left(-\lambda \frac{x}{r_{j+1}}\right)^{n}}{n !} b_{i}^{(j)}(n, n) .
$$

For $j=0$, we get from relation (4),

$$
b_{i}^{(0)}(n, 0)=\left(P_{B_{0} B_{0}}^{n} \mathbb{1}_{B_{0}}\right)(i) \mathbf{1}_{\left\{i \in B_{0}\right\}} .
$$

For $j=1, \ldots, v-1$, we get

$$
F_{i}\left(-\frac{x}{r_{j}}, x\right)=\lim _{t \rightarrow \frac{-x}{r_{j}}, t<\frac{-x}{r_{j}}} F_{i}(t, x)+\left(e^{-A_{B_{j} B_{j}} \frac{x}{r_{j}}} \mathbb{1}_{B_{j}}\right)(i) \mathbf{1}_{\left\{i \in B_{j}\right\}}
$$

It follows that, from relation (4),

$$
b_{i}^{(j)}(n, 0)=b_{i}^{(j-1)}(n, n)+\left(P_{B_{j} B_{j}}^{n} \mathbb{1}_{B_{j}}\right)(i) \mathbf{1}_{\left\{i \in B_{j}\right\}} .
$$

that is,

$$
\begin{aligned}
b_{B_{j}}^{(j)}(n, 0) & =b_{B_{j}}^{(j-1)}(n, n)+P_{B_{j} B_{j}}^{n} \mathbb{1}_{B_{j}} \\
b_{B_{l}}^{(j)}(n, 0) & =b_{B_{l}}^{(j-1)}(n, n) \text { for } l \neq j
\end{aligned}
$$

Last we consider the case where $j=v-1$, that is when $t \in\left[\frac{-x}{r_{v-1}},+\infty\right)$. In this case, since $r_{j+1}^{+}=0$, we get when $x \longrightarrow 0$, with $x>0, p_{j} \longrightarrow 1$ and so

$$
\lim _{x \rightarrow 0, x>0} F_{i}(t, x)=\sum_{n=0}^{\infty} e^{-\lambda t} \frac{(\lambda t)^{n}}{n !} b_{i}^{(v-1)}(n, n) .
$$

It follows from the initial condition expressed in Theorem 1 that

$$
b_{i}^{(v-1)}(n, n)=1 \text { if } d_{i} \leq 0,
$$

that is

$$
b_{B_{l}}^{(v-1)}(n, n)=\mathbb{1}_{B_{l}} \text { for } l=0, \ldots, v-1 .
$$




\section{Appendix B. Proof of Theorem 4}

a) These inequalities are immediate since the relation (7) and (8) are convex combinations of the vectors $b_{B_{l}}(n, k)$ that initially have their entries equal to 0 or 1 .

b) The relation is immediate for $n=0$ since we have $b_{B_{l}}(1,1) \geq 0=b_{B_{l}}(0,0)$ for $l=v, \ldots, m$ and $b_{B_{l}}(1,1)=b_{B_{l}}(0,0)=\mathbb{1}_{B_{l}}$ for $l=0, \ldots, v-1$.

Suppose the relation is satisfied at level $n-1, n \geq 1$, that is suppose that for all $l=0, \ldots, m$ and $0 \leq k \leq n$, we have $b_{B_{l}}(n, k) \geq b_{B_{l}}(n-1, k-1)$.

For $l=0, \ldots, v-1$, we have $b_{B_{l}}(n+1, n+1)=b_{B_{l}}(n, n)=\mathbb{1}_{B_{l}}$ which means that the relation is satisfied at level $n$ for $k=n$. Suppose the relation is satisfied at level $n$ for the integer $k+1$, that is suppose that we have $b_{B_{l}}(n+1, k+2) \geq b_{B_{l}}(n, k+1)$. Let us define $p=\frac{r_{l}-r_{v-1}}{r_{l}}$. We have $p \in[0,1]$. Using the relation $(8)$, we get

$$
\begin{aligned}
b_{B_{l}}(n+1, k+1)-b_{B_{l}}(n, k)= & p\left(b_{B_{l}}(n+1, k+2)-b_{B_{l}}(n, k+1)\right) \\
& +(1-p) \sum_{i=0}^{m} P_{B_{l} B_{i}}\left(b_{B_{i}}(n, k+1)-b_{B_{i}}(n-1, k)\right) \\
\geq 0, &
\end{aligned}
$$

from the recurrence hypothesis.

For $l=v, \ldots, m$, we have $b_{B_{l}}(n+1,1) \geq 0=b_{B_{l}}(n, 0)$ which means that the relation is satisfied at level $n$ for $k=0$. Suppose the relation is satisfied at level $n$ for the integer $k-1$, that is, suppose that we have $b_{B_{l}}(n+1, k) \geq b_{B_{l}}(n, k-1)$. Let us define $q=\frac{r_{l}}{r_{l}-r_{v-1}}$. We have $q \in[0,1]$. Using the relation (8), we get

$$
\begin{aligned}
b_{B_{l}}(n+1, k+1)-b_{B_{l}}(n, k)= & q\left(b_{B_{l}}(n+1, k)-b_{B_{l}}(n, k-1)\right) \\
& +(1-q) \sum_{i=0}^{m} P_{B_{l} B_{i}}\left(b_{B_{i}}(n, k)-b_{B_{i}}(n-1, k-1)\right) \\
\geq 0, &
\end{aligned}
$$

from the recurrence hypothesis. 
c) The relation is immediate for $n=0$ since we have $b_{B_{l}}(1,0)=b_{B_{l}}(0,0)=0$ for $l=v, \ldots, m$ and $b_{B_{l}}(1,0) \leq b_{B_{l}}(0,0)=\mathbb{1}_{B_{l}}$ for $l=0, \ldots, v-1$.

Suppose the relation is satisfied at level $n-1, n \geq 1$, that is suppose that for all $l=0, \ldots, m$ and $0 \leq k \leq n-1$, we have $b_{B_{l}}(n, k) \leq b_{B_{l}}(n-1, k)$.

For $l=0, \ldots, v-1$, we have $b_{B_{l}}(n+1, n) \leq b_{B_{l}}(n, n)=\mathbb{1}_{B_{l}}$ which means that the relation is satisfied at level $n$ for $k=n$. Suppose the relation is satisfied at level $n$ for the integer $k+1$, that is suppose that we have $b_{B_{l}}(n+1, k+1) \leq b_{B_{l}}(n, k+1)$. Let us define $p=\frac{r_{l}-r_{v-1}}{r_{l}}$. We have $p \in[0,1]$. Using the relation (8), we get

$$
\begin{aligned}
b_{B_{l}}(n+1, k)-b_{B_{l}}(n, k)= & p\left(b_{B_{l}}(n+1, k+1)-b_{B_{l}}(n, k+1)\right) \\
& +(1-p) \sum_{i=0}^{m} P_{B_{l} B_{i}}\left(b_{B_{i}}(n, k)-b_{B_{i}}(n-1, k)\right) \\
\leq 0, &
\end{aligned}
$$

from the recurrence hypothesis.

For $l=v, \ldots, m$, we have $b_{B_{l}}(n+1,0)=b_{B_{l}}(n, 0)=0$ which means that the relation is satisfied at level $n$ for $k=0$. Suppose the relation is satisfied at level $n$ for the integer $k-1$, that is suppose that we have $b_{B_{l}}(n+1, k-1) \leq b_{B_{l}}(n, k-1)$. Let us define $q=\frac{r_{l}}{r_{l}-r_{v-1}}$. We have $q \in[0,1]$. Using the relation $(7)$, we get

$$
\begin{aligned}
b_{B_{l}}(n+1, k)-b_{B_{l}}(n, k)= & q\left(b_{B_{l}}(n+1, k-1)-b_{B_{l}}(n, k-1)\right) \\
& +(1-q) \sum_{i=0}^{m} P_{B_{l} B_{i}}\left(b_{B_{i}}(n, k-1)-b_{B_{i}}(n-1, k-1)\right) \\
\leq 0, &
\end{aligned}
$$

from the recurrence hypothesis.

d) directly follows from b) and c).

e) directly follows from c) and d).

f) From inequality b), we deduce that for every $i \in S$, the sequence $b_{i}(n, n)$ is increasing. Moreover, from inequality a), we have $b_{i}(n, n) \leq 1$ so, the sequence $b_{i}(n, n)$ converges 
when $n$ goes to infinity. For every $i \in S$, we denote by $l_{i}$ the limit of the sequence $b_{i}(n, n)$. We then have

$$
F_{i}(t, 0)=\sum_{n=0}^{\infty} e^{-\lambda t} \frac{(\lambda t)^{n}}{n !} b_{i}(n, n) \longrightarrow l_{i} \text { when } t \longrightarrow \infty
$$

In another hand, since we have assumed that the stability condition $\sum_{i \in S} d_{i} \pi_{i}<0$ is satisfied, we have $F_{i}(t, 0) \longrightarrow 1$ when $t \longrightarrow \infty$. Thus, we conclude that for every $i \in S, l_{i}=1$.

\section{References}

[1] D. Anick, D. Mitra, and M. M. Sondhi. Stochastic theory of a data-handling system with multiple sources. Bell System Tech. J., 61(8):1871-1894, 1982.

[2] H. Kobayashi and Q. Ren. A mathematical theory for transient analysis of communication networks. IEICE Transactions on communications, E75-B(12):1266-1276, 1992.

[3] A. Narayanan and V. G. Kulkarni. First passages times in fluid models with an application to two priority fluid systems. In Proceedings of IPDS'96, Urbana-Champaign, Illinois, USA, pages 166-175, September 1996.

[4] Q. Ren and H. Kobayashi. Transient solutions for the buffer behavior in statistical multiplexing. Perf. Eval., 23:65-87, 1995.

[5] J. W. Roberts, U. Mocci, and J. Virtamo, editors. Broadband Network Teletraffic. Performance evaluation and design of broadband multiservice networks. Lecture Notes in Computer Science 1155, Springer, 1995. Final report of the COST 242 action.

[6] B. Sericola. Transient analysis of stochastic fluid models. Performance Evaluation, 32 (1998) 245-263.

$\mathrm{RR} \mathrm{n}^{\circ} 4057$ 
Unité de recherche INRIA Lorraine, Technopôle de Nancy-Brabois, Campus scientifique, 615 rue du Jardin Botanique, BP 101, 54600 VILLERS LÈS NANCY

Unité de recherche INRIA Rennes, Irisa, Campus universitaire de Beaulieu, 35042 RENNES Cedex

Unité de recherche INRIA Rhône-Alpes, 655, avenue de l'Europe, 38330 MONTBONNOT ST MARTIN

Unité de recherche INRIA Rocquencourt, Domaine de Voluceau, Rocquencourt, BP 105, 78153 LE CHESNAY Cedex

Unité de recherche INRIA Sophia-Antipolis, 2004 route des Lucioles, BP 93, 06902 SOPHIA-ANTIPOLIS Cedex

Éditeur

INRIA, Domaine de Voluceau, Rocquencourt, BP 105, 78153 LE CHESNAY Cedex (France)

http://www.inria.fr

ISSN 0249-6399 